\title{
ADDITIVE SET FUNCTIONS IN EUCLIDEAN SPACE. II
}

\section{BY}

\author{
C. A. ROGERS and S. J. TAYLOR \\ London
}

\section{Introduction}

In our previous paper [11], we discussed various decompositions of additive set functions in Euclidean space. Our main object was to show how a system of Hausdorff measures could be used to analyse a given set function, as far as is possible, into components, which were uniform in a certain sense. In the present work, we use the results of a series of papers $[5,8,9,12,13]$ to correct and extend some of the results obtained in [11].

We continue to restrict our attention to the system $\mathcal{I}$ of those continuous completely additive set functions $F$, having a finite value $F(E)$ for every set $E$ in the field $\mathcal{B}$ of all Borel subsets of a fixed closed rectangle $I_{0}$ in $k$-space. It is clear that the analysis extends immediately to $\sigma$-finite set functions, defined for Borel sets in Euclidean $k$-space.

In the first three sections of [11], we worked with a single Hausdorff measure function $h(t)$, and we obtained a unique decomposition of a set function $F$ of $\mathfrak{I}$ into three components, one strongly continuous with respect to $h$-measure, one, not only absolutely continuous with respect to $h$-measure, but also concentrated on a set of $\sigma$-finite $h$-measure, and one concentrated on a set of zero $h$-measure. The extensions and refinements of this work, which we made in [13] will be vital for the sequel.

In the last three sections of [11] we introduced a system $\mathcal{L}$ of Hausdorff measure functions $f(t)$, which was totally ordered by the relation $\prec$, defined by:

$$
f \prec g, \text { if } g(t) / f(t) \rightarrow 0, \quad \text { as } t \rightarrow+\mathbf{0} \text {. }
$$

We first studied the special case, when $\mathcal{L}$ is the system of functions

$$
t^{\alpha} \quad(0<\alpha \leqslant k)
$$


and then turned to the case when $\mathcal{L}$ is maximal. In these studies, we analysed a setfunction $F$ of $\mathcal{I}$ into a sequence of components, corresponding to certain sections of $\mathcal{L}$, and a residual component with a "continuous $\mathcal{L}$-dimension spectrum". While our main theorems (Theorems 3 and 6) are correct, the second of them (Theorem 6) cannot be regarded as satisfactory. Unfortunately, there is an error in the proof, that $F_{1}^{(s)}$ is continuous on the right, given in Lemma 10. The faulty lemma led to our introducing an unsatisfactory definition for a set-function with a "continuous $\mathcal{L}$-dimension spectrum"; a set-function satisfying our conditions would not, as far as we know, necessarily have the essential property of having no component which should naturally be associated with a section of $\mathcal{L}$.

In this paper, we correct and extend the analysis we made previously. The results we obtain are rather more complicated than those they replace. We find that they depend essentially on the various properties of the system $\mathcal{L}$, and on the nature of the sections of $\mathcal{L}$ considered. We defer any detailed description, until after we have introduced the necessary concepts. The results may be described, in general terms, by saying that, provided that the system $\mathcal{L}$ has appropriate density properties, we can analyse a set function $F$ of $\mathfrak{I}$ into:

(a) a sequence of isolated components corresponding to functions $h$ of $\mathcal{L}$;

(b) a sequence of isolated components corresponding to sections of $\mathcal{L}$ having no countable basis;

(c) a residual set function having no component belonging to any function or section of $\mathcal{L}$.

We give an example to show that the components corresponding to sections of $\mathcal{L}$ having no countable basis may be non-zero; but we have to leave unanswered some interesting questions concerning these components.

\section{Scales of functions}

Let $\mathcal{H}$ be the system of all positive functions $h(t)$, defined for $t>0$, continuous monotonic increasing, and with $\lim _{t \rightarrow+0} h(t)=0$. When $h$ and $g$ belong to $\mathcal{H}$, we write

if

and

if

$$
h \sim g,
$$$$
0<\liminf _{t \rightarrow+0} g(t) / h(t) \leqslant \limsup _{t \rightarrow+0} g(t) / h(t)<+\infty \text {; }
$$

$$
h \prec g \text {, }
$$

$$
\lim _{t \rightarrow+0} g(t) / h(t)=0
$$


We say that $h$ and $g$ in $\boldsymbol{H}$ are comparable, if

$$
h \prec g, \quad \text { or } \quad h \sim g, \quad \text { or } \quad g<h \text {; }
$$

and that they are monotonically comparable if, in addition, the ratio $h(t) / g(t)$ is monotonic for all sufficiently small positive $t$.

We list some properties, introduced in [5] and [8], which may hold for a subset $\mathcal{L}$ of $\boldsymbol{H}$.

$\left(\mathrm{P}_{1}\right)$ If $g, h \in \mathcal{L}$, then $g$ and $h$ are comparable.

( $\left.\mathrm{P}_{1}^{*}\right)$ If $g, h \in \mathcal{L}$, then $g$ and $h$ are monotonically comparable.

$\left(\mathrm{P}_{2}\right)$ The function $t^{k}$ is in $L$.

$\left(\mathrm{P}_{3}\right)$ If $g, h \in \mathcal{L}$ and $\alpha, \beta$ are real numbers, and $g^{\alpha} h^{\beta} \in \mathcal{H}$, then $g^{\alpha} h^{\beta} \in \mathcal{L}$.

$\left(\mathrm{P}_{4}\right)$ If $g, h \in \mathcal{L}$ and $g \neq h$, then $g$ is not equivalent to $h$.

$\left(\mathrm{P}_{5}\right)$ If $h \in \mathcal{H}$ and $h$ is comparable with each element of $\mathcal{L}$, then $h$ is equivalent to at least one element of $\mathcal{L}$.

$\left(\mathrm{P}_{6}\right)$ If $h \in \mathcal{H}$, there is at least one $g$ in $\mathcal{L}$ with $g \prec h$.

$\left(\mathrm{P}_{7}\right)$ If $h \in \mathcal{H}$ and $h \prec t^{k}$, there is at least one $g$ in $\mathcal{L}$ with $h<g<t^{k}$.

$\left(\mathbf{P}_{10}\right)$ If $l_{1}, l_{2}, \ldots, u_{1}, u_{2}, \ldots \in \mathcal{L}$ and $l_{1} \prec l_{2} \prec \ldots \prec l_{n} \prec \ldots \ldots \prec u_{m} \prec \ldots \prec u_{2} \prec u_{1}$, there is a $g$ in $\mathcal{L}$ with $l_{1} \prec l_{2} \prec \ldots \prec l_{n} \prec \ldots \prec g \prec \ldots \prec u_{m} \prec \ldots \prec u_{2} \prec u_{1}$.

$\left(\mathrm{P}_{11}\right)$ If $h \in \mathcal{H}$ and $l_{1}, l_{2} \ldots \in \mathcal{L}$ and $l_{1} \prec l_{2} \prec \ldots \prec l_{n} \prec \ldots \prec h$, there is a $g$ in $\mathcal{L}$ with $l_{1} \prec l_{2} \prec \ldots \prec l_{n} \prec \ldots \prec g \prec h$.

These conditions do not correspond exactly with those given in [5] and [8], since there we were concerned with functions which might tend to 0 or to $+\infty$ or to a finite limit as the variable tended to $+\infty$; but the differences are trivial.

A set $\mathcal{L}$ of functions of $\mathcal{H}$ will be called a monotone scale of functions, if it satisfies $\left(\mathrm{P}_{1}\right),\left(\mathbf{P}_{1}^{*}\right),\left(\mathrm{P}_{2}\right)$ and $\left(\mathrm{P}_{3}\right)$. A scale with property $\left(\mathrm{P}_{4}\right)$ is irreducible; one with $\left(P_{5}\right)$ is maximal; one with $\left(P_{6}\right)$ and $\left(P_{7}\right)$ is dense; and one with $\left(P_{6}\right),\left(P_{7}\right),\left(P_{10}\right)$ and $\left(\mathrm{P}_{11}\right)$ is strongly dense.

The main result which we obtained with the help of P. Erdös in [5] and [8] is:

THEOREM A. The continuum hypothesis implies the existence of a monotone scale $\mathcal{L}$ which is irreducible, maximal and strongly dense.

Remark. It is also shown in [8] that every maximal scale $\mathcal{L}$ has the property $\left(\mathrm{P}_{10}\right)$, and the same simple proof shows that such a scale $\mathcal{L}$ has the property obtained from $\left(\mathrm{P}_{10}\right)$ by replacing one or both of the sequences $l_{1}, l_{2}, \ldots$, or $u_{1}, u_{2}, \ldots$ by a single element of $\mathcal{L}$. 
At this stage we require one further definition. Two subsets $L, R$ of an irreducible scale $\mathcal{L}$ are said to form a section of $\mathcal{L}$, if:

$L$ has no maximal element,

$$
L \cap R=\varnothing, \quad L \cup R=\mathcal{L},
$$$$
\text { and } h_{1} \in L, h_{2} \in R \text { implies } h_{1} \prec h_{2} \text {. }
$$

We return to study and classify the sections of strongly dense irreducible monotone scales in $\$ 5$.

\section{Bands associated with a section in a scale of measure functions}

Let $\mathcal{L}$ be an irreducible maximal scale of functions of $\mathcal{H}$. Let $s$ be a section of $\mathcal{L}$ into two sets $L_{s}, R_{s}$. In [11] we associated two pairs of complementary bands ( $\left.{ }^{1}\right)$ in $\mathcal{I}$ with the section $s$. Let $R_{s}^{\prime}$ denote the set obtained by removing from $R_{s}$ its least element, if it has one. Then as in [11] we introduce:

1. $\mathcal{C}_{s}$ is the class of set functions $F$ of $\mathcal{F}$, which are $s$-continuous; that is, those set functions such that $F(E)=0$, for any $E \in \mathcal{B}$, for which there is at least one $h \in \mathcal{L}_{s}$ with $h-m(E)=0$;

2. $S_{s}$ is the class of set functions $F$ of $\mathcal{F}$, which are $s$-singular; that is, those set functions, for which there is some $E_{0}$ in $\mathcal{B}$, such that

$$
F(E)=F\left(E \cap E_{0}\right),
$$

and $E_{0}=\bigcup_{i=1}^{\infty} E_{i}$, where, for each $E_{i}$, there is some $h_{i}$ in $L_{s}$, for which $h_{i}-$ $m\left(E_{i}\right)=0$;

3. $\mathcal{C}_{s}^{*}$ is the class of set functions $F$ of $\mathcal{F}$, which are strongly $s$-continuous; that is, those set functions such that, if $E \in \mathcal{B}$, and $h-m(E)=0$, for every $h \in R_{s}^{\prime}$, then $F(E)=0$;

4. $S_{s}^{*}$ is the class of set functions $F$ of $\mathcal{F}$, which are almost $s$-singular; that is, those set functions, for which there is some $E_{0}$ in $\mathcal{B}$, such that (1) holds, and $h-m\left(E_{0}\right)=0$, for every $h$ in $R_{s}^{\prime}$.

In $[11]$, we showed that $\mathcal{C}_{s}, S_{s}$ and $\mathcal{C}_{s}^{*}, S_{s}^{*}$ are pairs of complementary bands in $\mathcal{F}$. In order to obtain a more complete analysis, we now define two further classes:

5. $\mathcal{C}_{s}^{* *}$ is the class of set functions $F$ of $\mathcal{I}$, which are hyper $s$-continuous; that is, those set functions which can be expressed as $F=\sum_{i=1}^{\infty} F_{i}$, with $\sum_{i=1}^{\infty}\left|F_{i}\right|\left(I_{0}\right)$ convergent, and each $F_{i}$ being $h_{i}$-continuous, for some $h_{i}$ in $R_{s}^{\prime}$;

(1) This concept is explained later in this section. 
6. $S_{s}^{* *}$ is the class of set functions $F$ of $\mathcal{I}$, which are hypo $s$-singular; that is, for each $h$ in $R_{s}^{\prime}$, there is a set $E_{0}$ in $\mathcal{B}$, such that $h-m\left(E_{0}\right)=0$, and (1) is satisfied.

The system $\mathcal{T}$ of sections $s$ of $\mathcal{L}$ is ordered in a natural way; we write $s<t$, if the corresponding left-hand sets $L_{s}$ and $L_{t}$ satisfy $L_{s} \subset L_{t}$, the inclusion being proper. It is clear, from the definitions, that, for any sections $u, s, t$, of $\mathcal{T}$, with $t<s<u$, we have

$$
\left.\begin{array}{l}
\mathcal{C}_{u} \subset C_{s}^{* *} \subset C_{s}^{*} \subset C_{s} \subset C_{t}^{* *} ; \\
S_{u} \supset S_{s}^{* *} \supset S_{s}^{*} \supset S_{s} \supset S_{t}^{* *} .
\end{array}\right\}
$$

Our first object is to prove that $\mathcal{C}_{s}^{* *}$ and $\boldsymbol{S}_{s}^{* *}$ form a pair of complementary bands in $\mathcal{F}$. This could be done directly for the space $\mathcal{F}$, but we prefer to prove a more general theorem about collections of bands in a UMB-lattice, and then obtain our results as a particular case. To this end, we recall the concept of a Banach lattice, with a uniformly monotone norm, and show that $\mathcal{F}$ is such a lattice.

As explained in [11], $\mathcal{F}$ is a vector lattice, that is a partially ordered linear space with a lattice structure. Further, $\mathcal{F}$ is conditionally complete: that is, every subset $\mathcal{U} \subset \mathcal{F}$, which is bounded above, has a least upper bound $F_{0}$ in $\mathcal{F}$. If $R$ is any conditionally complete vector lattice, we call a subset $\mathcal{U}$ of $\mathcal{R}$ a band, if:

(i) $\mathcal{U}$ is a vector subspace of $\mathcal{R}$;

(ii) if $G \in \mathcal{U}$, then every $F$ of $R$ with $|F| \leqslant|G|$ is also in $\mathcal{U}$;

(iii) $U$ is a sublattice of $R$, which is conditionally complete; that is, every subset $\vartheta$ of $\mathcal{U}$, which is bounded above in $R$, has its least upper bound in $R$ lying in $u$.

Here, in (ii), the element $|F|$ is defined(1) by

$$
|F|=F \cup\{-F\} \text {. }
$$

In a slightly more general context, Birkhoff ([1] p. 232) calls such a set a closed $l$-ideal.

If a vector lattice is also a Banach space, with the same vector structure, and with a norm related to the lattice structure by the condition that,

$$
\text { if }|F| \leqslant|G| \text {, then }\|F\| \leqslant\|G\|,
$$

it is called a Banach lattice (see [l]). It is easy to verify that $\mathcal{F}$ is a Banach lattice, under the norm

$$
\|F\|=|F|\left(I_{0}\right)
$$

(1) Note added to proof. The same symbols have been used for the lattice operations of join and meet for as the set operations of union and intersection; but this should cause no confusion. 
Following Birkhoff [1], we call a norm in a Banach lattice uniformly monotone when, given $\varepsilon>0$, one can find $\delta>0$, so that if $F \geqslant 0, G \geqslant 0$, and $\|F\|=1$, then $\|F+G\| \leqslant\|F\|+\delta$ implies $\|G\| \leqslant \varepsilon$. It is clear that $\mathcal{F}$ satisfies this condition, for, in $\mathcal{F}$, if $F \geqslant 0, G \geqslant 0$, then

$$
\|F+G\|=F\left(I_{0}\right)+G\left(I_{0}\right)=\|F\|+\|G\| .
$$

Any Banach lattice, which is a conditionally complete lattice, and which has a uniformly monotone norm, will be called a UMB-lattice.

In [11] we used some general theorems about conditionally complete lattices, which we now repeat for reference purposes. We recall that two elements $F, G$ in a vector lattice are said to be complementary, if

$$
|F| \cap|G|=0
$$

THEOREM B. If $\boldsymbol{R}$ is a conditionally complete vector lattice, and $\mathcal{U}$ is a subset of $\boldsymbol{R}$, the set $\vartheta$ of all elements of $\mathcal{R}$, which are complementary to every element of $\mathcal{U}$, is a band in $\boldsymbol{R}$. If $\mathcal{U}$ is also a band, then $\mathcal{U}$ consists of all the elements of $\mathfrak{R}$ which are complementary to every element of $\vartheta$.

This theorem follows almost immediately from the definitions; see N. Bourbaki [3], or S. Bochner and R. S. Phillips [2]. If $\mathcal{U}$ and $\vartheta$ are bands in $R$, each consisting of all the elements in $R$ complementary to all the elements of the other, we say that they are complementary bands in $\mathcal{R}$. The following fundamental decomposition theorem follows:

THEOREM C. Given two complementary bands $\mathcal{U}_{1}, \mathcal{U}_{2}$ in a conditionally complete vector lattice $\mathcal{R}$, each element $\boldsymbol{F}$ of $\boldsymbol{R}$ can be expressed uniquely as

$$
F=F_{1}+F_{2},
$$

where

$$
F_{1} \in \mathcal{U}_{1} \text { and } F_{2} \in \mathcal{U}_{2} \text {. }
$$

Further

$$
|F|=\left|F_{1}\right|+\left|F_{2}\right| \text {. }
$$

The existence and uniqueness of the decomposition is essentially due to Riesz [7]. If $F_{1}, F_{2}$ are complementary elements of any vector lattice, then

$$
\left|F_{1}+F_{2}\right|=\left|F_{1}\right|+\left|F_{2}\right| \text {. }
$$

(This result is well known: a proof can be easily constructed using the identities on page 19 of Bourbaki [3].) 
THEOREM D. Any band in a conditionally complete Banach lattice is closed under the metric.

Proof. Suppose that $\mathcal{U}$ is a band and that $\vartheta$ is the complementary band. Let $F$ be any limit point of $\mathcal{U}$. Then there is a sequence $F_{1}, F_{2}, \ldots$ in $U$ converging in norm to $F$. Let $G$ belong to $\vartheta$. Then as $G, F_{i}$ belong to complementary bands,

$$
\begin{aligned}
|G| \cap|F| & =|| G|\cap| F|-| G|\cap| F_{i}|| \\
& \leqslant|| F|-| F_{i}|| \leqslant\left|F-F_{i}\right|,
\end{aligned}
$$

so that

$$
\||G| \cap|F|\| \leqslant\left\|F-F_{i}\right\|
$$

for all $i$. Hence $|G| \cap|F|=0$ and $F$ is complementary to $G$. Thus $F$ belongs to the band complementary to $\mathfrak{V}$, i.e., to $\mathcal{U}$. Thus $\mathcal{U}$ is closed under the metric.

We also need

THEOREM E. If a set $\mathcal{U}$ in a UMB-lattice $\boldsymbol{R}$ satisfies the conditions:

(i) $\mathcal{U}$ is a vector subspace of $R$, which is closed under the metric;

(ii) if $G \in \mathcal{U}$, then every $F$ of $\boldsymbol{R}$ with $|F| \leqslant|G|$ also belongs to $\mathcal{U}$;

then it is a band in $R$.

Remark. In Birkhoff's terminology, this says that an $l$-ideal in a UMB-lattice, which is closed under the metric, is a closed $l$-ideal.

Proof. It suffices to prove that $\mathcal{U}$ is conditionally complete. Now, by (i), the set $\mathcal{U}$ is a sub-Banach space of $R$. Further, if $F$ and $G$ belong to $U$, then, by (ii), we have $|F|$ and $|G|$ in $\mathcal{U}$. Thus $|F|+|G| \in \mathcal{U}$ by (i). But

so that by (ii) we have

$$
\begin{aligned}
& |F \cap G| \leqslant|F|+|G|, \\
& |F \cup G| \leqslant|F|+|G|
\end{aligned}
$$

$$
F \cap G \in \mathcal{U} \text { and } F \cup G \in \mathcal{U}
$$

Thus $\mathcal{U}$ is a sub-lattice of $\mathcal{R}$.

Now suppose that $\vartheta$ is a subset of $u$ which is bounded above by an element $R_{0}$ of $R$. Let $V_{0} \in \mathcal{W}$ and consider the system $\mathcal{W}$ of finite joins

$$
V_{0} \cup V_{1} \cup \ldots \cup V_{k} \quad(k \geqslant 0)
$$

$\mathcal{W}$ is a directed set contained in $\mathcal{U}$ with

$$
V_{0} \leqslant W \leqslant R_{0}
$$


for all $W \in \mathcal{W}$. Hence, by [1], Theorem 11, page 249, the directed set $W$ converges metrically to some element, $U_{1}$ say, of $R$. As $\mathcal{W} \subset \mathcal{U}$, and $\mathcal{U}$ is closed under the metric, $U_{1} \in \mathcal{U}$. Further $U_{1}$ is the least upper bound of $\mathcal{V}$ in $\boldsymbol{R}$. Consequently $\mathcal{U}$ is conditionally complete, as required.

These two results enable us to prove

THEOREM F. Suppose $\boldsymbol{R}$ is a UMB-lattice and $\mathcal{U}_{\alpha}$ is a band in $\boldsymbol{R}$ for each $\alpha$ in an index set J. Then

$$
\bigcap_{x \in y} u_{\alpha}
$$

is a band in $\boldsymbol{R}$. Further, the closure under the metric of the vector space generated by the union

$$
\bigcup_{\alpha \in \mathcal{Y}} U_{\alpha}
$$

is a band in $R$, and is the least band in $R$ containing this union.

Remark. We shall only need the special case when the bands $\mathcal{U}_{\alpha}$ with $\alpha \in \mathcal{J}$ are nested (i.e., are totally ordered by the relation of set inclusion). In this case, the result follows almost immediately from Theorems $B, D$ and $E$; but the general result seems to be sufficiently interesting to justify its special proof.

Proof. It follows immediately from the definitions that

is a band in $\boldsymbol{R}$.

$$
\bigcap_{\alpha \in \supset} u_{\alpha}
$$

Let $Q$ be the closure under the metric of the vector space generated by the union

$$
\bigcup_{\alpha \in \mathcal{J}} U_{\alpha}
$$

It follows, from Theorem $\mathrm{D}$, that any band in $R$, containing this union, must also contain $Q$. Hence it suffices to prove that $Q$ is a band in $R$. But $Q$ satisfies the condition (i) of Theorem E. So it suffices to prove that $Q$ satisfies the condition (ii) of Theorem E.

We first prove that, if $\mathcal{U}$ and $\mathcal{v}$ are two vector spaces in $\boldsymbol{R}$ satisfying condition (ii) of Theorem $\mathrm{E}$, then the vector space $\mathcal{W}$ generated by the union of $\mathcal{U}$ and $\vartheta$ also satisfies this condition. Suppose that $W \in W$ and $F$ in $R$ satisfies $\left|F^{\prime}\right| \leqslant|W|$. Then $W=U_{1}+V_{1}$ with $U_{1} \in \mathcal{U}$ and $V_{1} \in \mathcal{\vartheta}$. Further

and

$$
F^{+} \leqslant|F| \leqslant\left|U_{1}+V_{1}\right| \leqslant\left|U_{1}\right|+\left|V_{1}\right|
$$

$$
U_{2}=\left|U_{1}\right| \in \mathcal{U}, \quad V_{2}=\left|V_{1}\right| \in \mathcal{\vartheta} \text {. }
$$


Hence

Write

Then

Also

and

so that

$$
F^{+}=F^{+} \cap\left(U_{2}+V_{2}\right)=U_{2}+\left(F^{+}-U_{2}\right) \cap V_{2} .
$$

$$
U_{3}=\left\{\left(F^{+}-U_{2}\right) \cap V_{2}\right\} \cap 0
$$$$
V_{3}=\left\{\left(F^{+}-U_{2}\right) \cap V_{2}\right\} \cup 0 \text {. }
$$

$$
F^{+}==U_{2}+U_{3}+V_{3} \text {. }
$$

$$
0 \leqslant-U_{3}=\left\{\left(U_{2}-F^{+}\right) \cup\left(-V_{2}\right)\right\} \cup 0 \leqslant U_{2} \text {, }
$$

$$
0 \leqslant V_{3}=\left\{\left(F^{+}-U_{2}\right) \cap V_{2}\right\} \cup 0 \leqslant V_{2},
$$

$$
U_{3} \in \mathcal{U}, \quad V_{3} \in \mathcal{V} \text { and } F^{+} \in \mathcal{W} \text {. }
$$

Similarly $F^{-} \in W$ and so does $F=F^{+}+F^{-}$.

Applying this result inductively we see that the vector space generated by the union of any finite collection of the sets $U_{\alpha}$ with $\alpha \in \mathcal{J}$ satisfies the condition (ii) of Theorem E.

Now suppose that $G \in Q$, and that $F$ in $R$ satisfies $|F| \leqslant|G|$. As $G \in Q$, we have

$$
G=\lim _{i \rightarrow \infty} G_{i}
$$

where for each $i$,

$$
G_{i}=\sum_{k=1}^{j(i)} G_{i k}
$$

$$
\begin{array}{cl}
G_{i k} \in \mathcal{U}_{\alpha(i, k)} & (k=1,2, \ldots, j(i)), \\
\alpha(i, k) \in \mathcal{J} & (k=1,2, \ldots, j(i)) .
\end{array}
$$

Since $0 \leqslant F^{+} \leqslant|G|, 0 \leqslant-F^{-} \leqslant|G|$, we have

$$
\begin{aligned}
& \qquad \begin{aligned}
\left|F-F^{+} \cap\right| G_{i}\left|+\left(-F^{-}\right) \cap\right| G_{i}|| & =\left|F^{+} \cap\right| G\left|-F^{+} \cap\right| G_{i}\left|-\left(-F^{-}\right) \cap\right| G\left|-\left(-F^{-}\right) \cap\right| G_{i}|| \\
& \leqslant 2|| G|-| G_{i}|| \leqslant 2\left|G-G_{i}\right| .
\end{aligned} \\
& \text { So } \quad\left\|F-F^{+} \cap\left|G_{i}\right|+\left(-F^{-}\right) \cap\left|G_{i}\right|\right\| \leqslant 2\left\|G-G_{i}\right\| .
\end{aligned}
$$

So

Thus $F$ is the limit in norm of the sequence

$$
H_{i}=F^{+} \cap\left|G_{i}\right|-\left(-F^{-}\right) \cap\left|G_{i}\right| \quad(i=1,2, \ldots) .
$$

But, for each $i$, the vector space generated by the sets

$$
\mathcal{U}_{\alpha(i, k)} \quad(k=1,2, \ldots, j(i)),
$$

satisfies condition (ii) of Theorem E. 
So

$$
F^{+} \cap\left|G_{i}\right| \text { and }\left(-F^{-}\right) \cap\left|G_{i}\right|
$$

lie in this vector space and thus belong to $Q$. Consequently $H_{i}, i=1,2, \ldots$ anc $F=\lim _{i \rightarrow \infty} H_{i}$ belong to the closed vector space $Q$. This completes the proof.

We now return to the study of the space $\mathcal{I}$ of additive set functions. We prove

THEOREM G. For any section $s$ of $\mathcal{L}$, the sets $\mathcal{C}_{s}^{* *}$ and $\mathcal{S}_{s}^{* *}$ are complementary bands in $\mathcal{F}$.

Proof. Suppose $s$ is the section $L_{s}, R_{s}$ of $\mathcal{H}$. For each $h$ in $R_{s}^{\prime}$, the class $\mathrm{C}(h)$ of $h$-continuous set functions, and the class $S(h)$ of $h$-singular set functions form com. plementary bands in $\mathcal{F}$. So, by Theorem $F$, the set

is a band in $\mathcal{F}$.

$$
S_{s}^{* *}=\bigcap_{h \in R_{s}^{\prime}} S(h)
$$

Let $Q$ be the closure under the norm of the vector space generated by the union

$$
\bigcup_{h \in R_{s}^{\prime}} \mathcal{C}(h) \text {. }
$$

Now every element in this union is complementary to each element of $S_{s}^{* *}$. It follows that each element of $Q$ is also complementary to each element of $\boldsymbol{S}_{s}^{* *}$. But each element of $\mathcal{F}$, which is complementary to each element of $Q$, lies in $\mathcal{S}(h)$ for all $h \in R_{s}^{\prime}$, and so lies in $S_{s}^{* *}$. Thus $S_{s}^{* *}$ is the band complementary to the set $Q$. But, by Theorem $\mathrm{F}$, the set $Q$ is a band in $\mathcal{F}$. Hence $\mathcal{S}_{s}^{* *}$ and $Q$ are complementary bands in $\mathcal{F}$, and it remains to identify $Q$ and $\mathcal{C}_{s}^{* *}$.

Since every $F$ of $\mathcal{F}$ of the form

$$
F=\sum_{i=1}^{\infty} F_{i}
$$

with $\sum_{i=1}^{\infty}\left\|F_{i}^{\prime}\right\|$ convergent, and with $F_{i} \in \mathcal{C}\left(h_{i}\right)$, for some $h_{i}$ in $R_{s}^{\prime}$, is clearly in $Q$, we have $\mathcal{C}_{s}^{* *} \subset \boldsymbol{Q}$.

Now, if $F \in Q$, we have

$$
F=\lim _{i \rightarrow \infty} G_{i}
$$

for some sequence $G_{1}, G_{2}, \ldots$ of set functions in the vector space generated by the union

$$
\bigcup_{h \in R_{s}^{\prime}} \mathcal{C}(h)
$$

Replacing $G_{1}, G_{2}, \ldots$ by a suitable sub-sequence $H_{1}, H_{2}, \ldots$, and writing $H_{0}=0$, we have 


$$
F=\sum_{i=1}^{\infty}\left(H_{i}-H_{i-1}\right)
$$

with

$$
\sum_{i=1}^{\infty}\left\|H_{i}-H_{i-1}\right\|
$$

convergent. Now, for each $i$,

$$
F_{i}=H_{i}-H_{i-1}
$$

lies in the vector space generated by the union (5), and so has a representation as a finite sum

$$
F_{i}=\sum_{k=1}^{j(i) \mid} F_{i k}
$$

with

$$
F_{i k} \in \mathrm{C}\left(h_{i k}\right) \quad(k=1,2, \ldots, j(i))
$$

and

$$
h_{i k} \in R_{s}^{\prime} \quad(k=1,2, \ldots, j(i)) .
$$

Thus $Q \supset C_{s}^{* *}$, and the theorem is proved.

\section{The first decomposition theorems}

As in section 3 , let $\mathcal{L}$ be an irreducible maximal scale of functions of $\mathcal{H}$. In this section, we show how a function $F$ of $\mathcal{F}$ can be expressed as a sum of components, associated with sections $s$ of the system $\mathcal{T}$ consisting of all sections of $\mathcal{L}$, and a residual component having what we shall call a continuous dimension spectrum. We first prove

THEOREM 1. Given any set function $F \in \mathcal{F}$ and any section $s$ of $\mathcal{L}$, there is a unique decomposition

$$
F=F_{1}^{(s)}+F_{2}^{(s)}+F_{3}^{(s)}+F_{4}^{(s)}
$$

where $F_{1}^{(s)}$ is hyper s-continuous, $F_{2}^{(s)}$ is strongly s-continuous and hypo s-singular, $F_{3}^{(s)}$ is s-continuous and almost s-singular, and $F_{4}^{(s)}$ is s-singular. Further

$$
|F|=\left|F_{1}^{(s)}\right|+\left|F_{2}^{(s)}\right|+\left|F_{3}^{(s)}\right|+\left|F_{4}^{(s)}\right|
$$

Remark. The decomposition of this theorem is apparently finer than that of Theorem 5 of $[11]$; the set function $F_{1}^{(s)}+F_{2}^{(s)}$ above corresponds to the first component of the decomposition given in [11]; but we know of no example with $F_{2}^{(s)} \neq 0$. Thus we have been unable to decide whether or not the class $C_{s}^{*} \cap S_{s}^{* *}$ is always void.

Note that, if $t^{k} \in L_{s}$, then necessarily

$$
F=F_{4}^{(s)}, \quad F_{1}^{(s)}=F_{2}^{(s)}=F_{3}^{(s)}=0 .
$$

See also the remark at the end of section 8 .

15-632918 Acta mathematica 109. Imprimé le 14 juin 1963. 
Proof. By the definitions, the results of [1], and Theorem G, the pairs $\left\{\mathrm{C}_{s}, S_{s}\right\}$, $\left\{\mathcal{C}_{s}^{*}, S_{s}^{*}\right\}$ and $\left\{\mathcal{C}_{s}^{* *}, S_{s}^{* *}\right\}$, are pairs of complementary bands in $\mathcal{F}$, and satisfy

$$
\begin{aligned}
& C_{s}^{* *} \subset C_{s}^{*} \subset C_{s}, \\
& S_{s}^{* *} \supset S_{s}^{*} \supset S_{s} .
\end{aligned}
$$

The result now follows, on applying Theorem $\mathrm{C}$ three times.

We now need to know how the decomposition of Theorem 1 depends on the section $s$ of $\mathcal{L}$. We can define left and right continuity in the norm topology of $\mathcal{F}$ relative to the order topology of $\mathcal{J}$. If $F(s)$ is a set function of $\mathcal{I}$ defined for $s$ in $\mathcal{J}$, we say that $F(s)$ is continuous on the right at $s$, if, for each $\varepsilon>0$, there is a $u$ in $\mathfrak{J}$, such that

$$
\|F(s)-F(t)\|<\varepsilon, \quad \text { for all } t \text { in } \mathcal{J} \text { with } s<t<u
$$

Continuity on the left is defined similarly, and the discontinuities on the left and right of a function with a simple jump discontinuity are defined in the natural way.

THEOREM 2. In the decomposition of Theorem 1,

$$
-\left\|F_{1}^{(s)}\right\| \text { and }\left\|F_{4}^{(s)}\right\|
$$

are monotonic increasing functions of $s$, the function $F_{1}^{(s)}$ is continuous on the right, and $F_{4}^{(s)}$ is continuous on the left. The sections $s$, where $-F_{1}^{(s)}$ and $F_{4}^{(s)}$ are discontinuous coincide, and at such a section $-F_{1}^{(s)}$ has a simple jump discontinuity on the left and $F_{4}^{(s)}$ has a simple jump discontinuity on the right, these discontinuities being equal and having the value

$$
F_{2}^{(s)}+F_{3}^{(s)}
$$

Remark 1. This result, as Lemma 10 of [11] was alleged to, follows from use of the methods of $\S 4$ of [11], but, in view of the lacuna we have found in the proof of Lemma 10, it seems best to give the proof in some detail.

Remark 2. Since $\|F\|=|F|\left(I_{0}\right)$ and $|F|$ is non-negative for all $F \in \mathcal{F}$, the fact that $-\left\|F_{1}^{(s)}\right\|$ and $\left\|F_{4}^{(s)}\right\|$ are monotonic increasing implies that $-\left|F_{1}^{(s)}\right|(E)$ and $\left|F_{4}^{(s)}\right|(E)$ are monotonic increasing for all $E \in \mathcal{B}$.

Proof. Suppose that $s$ and $t$ are sections of $\mathcal{L}$ with $s<t$. Let

$$
\begin{aligned}
& |F|=\left|F_{1}^{(s)}\right|+\left|F_{2}^{(s)}\right|+\left|F_{3}^{(s)}\right|+\left|F_{4}^{(s)}\right|, \\
& |F|=\left|F_{1}^{(t)}\right|+\left|F_{2}^{(t)}\right|+\left|F_{3}^{(t)}\right|+\left|F_{4}^{(t)}\right|
\end{aligned}
$$


be the corresponding decompositions of $|F|$, provided by Theorem 1 . Now, by the inclusion relations (2), the function $\left|F_{1}^{(t)}\right|$ is hyper $s$-continuous. Further, by Theorem 1 , we have a decomposition

$$
\left|F_{2}^{(t)}\right|+\left|F_{3}^{(t)}\right|+\left|F_{4}^{(t)}\right|=G_{1}^{(s)}+G_{2}^{(s)}+G_{3}^{(s)}+G_{4}^{(s)},
$$

where $G_{i}^{(s)}, i=1,2,3,4$, are all non-negative, and where $G_{1}^{(s)}$ is hyper $s$-continuous, $G_{2}^{(s)}$ is strongly $s$-continuous and hypo $s$-singular, $G_{3}^{(s)}$ is $s$-continuous and almost $s$-singular, and $G_{4}^{(s)}$ is $s$-singular. Since the decomposition of $|F|$ with respect to the section $s$ is unique, we have the identification

$$
\begin{aligned}
& \left|F_{1}^{(s)}\right|=\left|F_{1}^{(t)}\right|+G_{1}^{(s)}, \\
& \left|F_{i}^{(s)}\right|=G_{i}^{(s)} \quad(i=2,3,4) .
\end{aligned}
$$

Since $G_{1}^{(s)}$ is non-negative, we have

$$
\begin{aligned}
\left\|F_{1}^{(s)}\right\| & =\left|F_{1}^{(s)}\right|\left(I_{0}\right)=\left|F_{1}^{(t)}\right|\left(I_{0}\right)+G_{1}^{(s)}\left(I_{0}\right) \\
& \geqslant\left|F_{1}^{(t)}\right|\left(I_{0}\right)=\left\|F_{1}^{(t)}\right\|
\end{aligned}
$$

Thus - $\left\|F_{1}^{(s)}\right\|$ is monotonic increasing. Similarly $\left\|F_{4}^{(s)}\right\|$ is monotonic decreasing.

Now we study the behaviour of $F_{1}^{(t)}$, for $t$ in $\mathcal{J}$ to the right of a section $s \in \mathcal{J}$. The function $F_{1}^{(s)}$ itself is hyper s-continuous and so has a representation

$$
F_{1}^{(s)}=\sum_{i=1}^{\infty} G_{i}
$$

with $\sum_{i=1}^{\infty}\left\|G_{i}\right\|$ convergent, and with each $G_{i}$ being $h_{i}$-continuous, for some $h_{i}$ in the class $R_{s}^{\prime}$ associated with the section $s$. For each $\varepsilon>0$, we can choose a positive integer $k$, so that

$$
\sum_{i=k+1}^{\infty}\left\|G_{i}\right\|<\varepsilon
$$

Let

$$
h=\min \left\{h_{1}, h_{2}, \ldots, h_{k}\right\}
$$

Then $h \in R_{s}^{\prime}$.

If $R_{s}$ has no minimal element, then $R_{s}^{\prime}=R_{s}$ has no minimal element. On the other hand, if $R_{s}$ has a minimal element, then $R_{s}^{\prime}$ was formed by removing it, and it follows from the maximality of $\mathcal{L}$ that $R_{s}^{\prime}$ has no minimal element.

Thus we can choose $g$ in $R_{s}^{\prime}$, with $g \prec h$, and we shall still have elements $r$ in $R_{s}^{\prime}$, with $r \prec g$. Let $u$ be the section of $\mathcal{L}$ formed by taking the left class to be all the functions $r$ of $\mathcal{L}$ with $r \prec g$. Then $s<u$, and $g$ belongs to the right class of $u$. 
Consider any section $t$ in $T$, with

$$
s<t<u \text {. }
$$

As $g$ belongs to the right class of $t$, and

$$
g \prec h_{i} \quad(i=1,2, \ldots, k),
$$

the set function

$$
\sum_{i=1}^{k} G_{i}
$$

is hyper $t$-continuous. The set function

$$
H=\sum_{i=k+1}^{\infty} G_{i}
$$

has a decomposition in the canonical form

$$
H=H_{1}^{(t)}+H_{2}^{(t)}+H_{3}^{(t)}+H_{4}^{(t)} .
$$

The set function

$$
K=F_{2}^{(s)}+F_{3}^{(s)}+F_{4}^{(s)}
$$

is hypo $s$-singular and so $l$-singular for any $l$ in the modified right class $R_{s}^{\prime}$ of $s$. Taking $l$ to be in $R_{s}^{\prime}$ and also in the modified left class of $t$ we see that $K$ is $t$-singular. Thus comparing the decomposition

$$
F^{\prime}=\left\{\sum_{i=1}^{k} G_{i}+H_{1}^{(t)}\right\}+H_{2}^{(t)}+H_{\mathbf{5}}^{(t)}+\left\{H_{4}^{(t)}+K\right\}
$$

with the decomposition with respect to the section $t$ provided by Theorem 1 , we have

$$
F_{1}^{(t)}=\sum_{i=1}^{k} G_{i}+H_{1}^{(t)}
$$

Hence

$$
\left\|F_{1}^{(t)}-F_{1}^{(s)}\right\|=\left\|H_{2}^{(t)}+H_{3}^{(t)}+H_{4}^{(t)}\right\| \leqslant\|H\|<\varepsilon
$$

This proves the continuity of $F_{1}^{(t)}$ on the right at $t=s$.

But it also follows from the decomposition (6) that

$$
F_{4}^{(t)}=H_{4}^{(t)}+K
$$

Hence

$$
\left\|F_{4}^{(t)}-F_{2}^{(s)}-F_{3}^{(s)}-F_{4}^{(s)}\right\|=\left\|H_{4}^{(t)}\right\| \leqslant\|H\|<\varepsilon
$$

This proves that $F_{4}^{(t)}$ has a simple discontinuity $F_{2}^{(s)}+F_{3}^{(s)}$ on the right at $t=s$, or is continuous on the right at $t=s$ in the case when $F_{2}^{(s)}+F_{3}^{(s)}=0$.

We remark that, if it is possible to choose $h$ in $R_{s}^{\prime}$ so that

$$
h \prec h_{i} \quad(i=1,2,3, \ldots),
$$


then the above proof shows that

$$
\begin{gathered}
F_{1}^{(t)}=F_{1}^{(s)}, \quad F_{2}^{(t)}=0, \quad F_{3}^{(t)}=0, \\
F_{4}^{(t)}=F_{2}^{(s)}+F_{3}^{(s)}+F_{4}^{(s)}
\end{gathered}
$$

for all $t$ with $s<t \leqslant u$. Thus, in this case, $F_{1}^{(t)}$ and $F_{4}^{(t)}$ are constant and $F_{2}^{(t)}$ and $F_{3}^{(t)}$ vanish in an open interval to the right of $t=s$.

We now study the behaviour of $F_{4}^{(t)}$, for $t$ in $\mathcal{J}$. The function $F_{4}^{(s)}$ is itself $s$-singular. So $F_{4}^{(s)}$ is concentrated on a set $E_{0}$ in $\mathcal{B}$ and

$$
E_{0}=\bigcup_{i=1}^{\infty} E_{i}
$$

where, for each $i$ there is some $h_{i}$ in $L_{s}$ for which $h_{i}-m\left(E_{i}\right)=0$. Without loss of generality we may assume that the sets $E_{i}$ are disjoint Borel sets. Now, writing

$$
G_{i}(E)=F_{4}^{(s)}\left(E \cap E_{i}\right)
$$

we have

$$
F_{4}^{(s)}=\sum_{i=1}^{\infty} G_{i}
$$

and

$$
\sum_{i=1}^{\infty}\left\|G_{i}\right\|=\sum_{i=1}^{\infty}\left|F_{4}^{(s)}\right|\left(E_{i}\right)=\left\|F_{4}^{(s)}\right\|<+\infty
$$

The argument now parallels that used in the discussion of $F_{1}^{(t)}$. For each $\varepsilon>0$, we can choose a positive integer $k$ so that

Then

$$
\begin{gathered}
\sum_{i=k+1}^{\infty}\left\|G_{i}\right\|<\varepsilon . \\
h=\max \left\{h_{1}, h_{2}, \ldots, h_{k}\right\}
\end{gathered}
$$

belongs to $L_{s}$. Further $L_{s}$ has no maximal element. So we can construct a section $u$ in $\mathcal{J}$ with $u<s$ having a function $g$ with $h \prec g$ in its left class.

Consider any $t$ in $\mathfrak{J}$ with

$$
u<t<s \text {. }
$$

As $g$ belongs to the left class of $t$, and

$$
h_{i} \prec g \quad(i=1,2, \ldots, k),
$$

the set function

$$
\sum_{i=1}^{k} G_{i}
$$

is $t$-singular. The set function

$$
H=\sum_{i=\hat{k}+1}^{\infty} G_{i}
$$


has a canonical decomposition, say

$$
H=H_{1}^{(t)}+H_{2}^{(t)}+H_{3}^{(t)}+H_{4}^{(t)}
$$

with respect to the section $t$. The set function

$$
K=F_{1}^{(s)}+F_{2}^{(s)}+F_{3}^{(s)}
$$

is $s$-continuous. So $K$ is $l$-continuous for each $l \in L_{s}$. Since $t<s, K$ is $l$-continuous for at least one $l$ in $R_{t}^{\prime}$. Hence $K$ is hyper $t$-continuous. Comparing the decomposition

$$
F=\left\{H_{1}^{(t)}+K\right\}+H_{2}^{(t)}+H_{3}^{(t)}+\left\{H_{4}^{(t)}+\sum_{i=1}^{k} G_{i}\right\}
$$

with the decomposition of Theorem 1, we obtain the identification

$$
\begin{aligned}
& F_{1}^{(t)}=H_{1}^{(t)}+K, \\
& F_{2}^{(t)}=H_{2}^{(t)}, \\
& F_{3}^{(t)}=H_{3}^{(t)}, \\
& F_{4}^{(t)}=H_{4}^{(t)}+\sum_{i=1}^{k} G_{i} .
\end{aligned}
$$

It now follows, as above, that $F_{4}^{(t)}$ is continuous on the left at $t=s$, and that $F_{1}^{(t)}$ has a simple discontinuity $-F_{2}^{(s)}-F_{3}^{(s)}$ on the left at $t=s$, or is continuous on the left at $t=s$ in the case when $F_{2}^{(s)}+F_{3}^{(s)}=0$.

Further, if it is possible to choose $h$ in $L_{s}$ so that

$$
h_{i} \prec h \quad(i=1,2,3, \ldots)
$$

then

$$
F_{1}^{(t)}=F_{1}^{(s)}+F_{2}^{(s)}+F_{3}^{(s)}
$$

$$
F_{2}^{(t)}=0, \quad F_{3}^{(t)}=0, \quad F_{4}^{(t)}=F_{4}^{(s)}
$$

for all $t$ with $u \leqslant t<s$.

Combining the above results we see that we have completed the proof of Theorem 2.

Two new concepts are now necessary.

DEfINITION. If $s \in \mathcal{J}$ and $F \in \mathcal{F}$, then $F$ is said to have the exact $\mathcal{L}$-dimension $s$, if $F$ is s-continuous and hypo s-singular.

Definition. $A$ set function $F$ of $\mathcal{F}$ is said to have a diffuse $\mathcal{L}$-dimension spectrum, if there is no set function $G$ of $₹$ with $0<|G| \leqslant|F|$ having an exact $\mathcal{L}$-dimension. 
These definitions are intended to supersede the definitions given in section 6 of [1], which we now regard as being inappropriate. But the examples of section 5 of [1] are still relevant, and provide examples of set functions having exact $\mathcal{L}$-dimension $s$, and of a set function having a diffuse $\mathcal{L}$-dimension spectrum, on taking $\mathcal{L}$ to be any irreducible maximal scale of functions.

The following theorem characterizes the set functions, with a diffuse $\mathcal{L}$-dimension spectrum, in terms of their behaviour under decomposition.

THEOREM 3. A set function $F$ of $¥$ has a diffuse L-dimension spectrum, if, and only if, its decomposition

$$
F=F_{1}^{(t)}+F_{2}^{(t)}+F_{3}^{(t)}+F_{4}^{(t)}
$$

for $t$ in $\mathcal{J}$, satisfies one of the following equivalent conditions:

(1) $F_{1}^{(t)}$ is continuous for all $t$ in $\mathcal{J}$;

(2) $F_{2}^{(t)}=F_{3}^{(t)}=0$ for all $t$ in $\mathcal{J}$;

(3) $F_{4}^{(t)}$ is continuous for all $t$ in $\mathcal{T}$.

Proof. It follows immediately, from Theorem 2, that the three conditions are equivalent.

If the condition (2) fails, there is a $t$ in $\mathcal{T}$, with, either

$$
F_{2}^{(t)} \neq 0, \quad \text { or } \quad F_{3}^{(t)} \neq 0 \text {. }
$$

Then

$$
0<\left|F_{2}^{(t)}\right|+\left|F_{3}^{(t)}\right| \leqslant\left|F_{1}^{(t)}\right|+\left|F_{2}^{(t)}\right|+\left|F_{3}^{(t)}\right|+\left|F_{4}^{(t)}\right|=|F|,
$$

while $\left|F_{2}^{(t)}\right|+\left|F_{3}^{(t)}\right|$ is $t$-continuous and hypo $t$-singular. Hence $F$ does not have a diffuse $\mathcal{L}$-dimension spectrum.

On the other hand, if $F$ does not have a diffuse $\mathcal{L}$-dimension spectrum, there is a $t$ in $\mathfrak{T}$ and a $G$ in $\mathcal{F}$ with

$$
0<|G| \leqslant|F|
$$

$G$ being $t$-continuous and hypo $t$-singular. Now we can write

$$
|F|=|G|+|H|
$$

where $H=|F|-|G|$. Decomposing $|F|,|G|$ and $|H|$ with respect to the section $t$, and comparing the two resulting decompositions of $|F|$, we have

$$
\begin{aligned}
\left|F_{2}^{(t)}\right|+\left|F_{3}^{(t)}\right| & =\left|G_{2}^{(t)}\right|+\left|G_{3}^{(t)}\right|+\left|H_{2}^{(t)}\right|+\left|H_{3}^{(t)}\right| \\
& \geqslant\left|G_{2}^{(t)}\right|+\left|G_{3}^{(t)}\right|=|G|>0 .
\end{aligned}
$$

Thus the condition (2) fails. This completes the proof.

We are now in a position to state the main theorem of this section. 
THEOREM 4. Given any irreducible maximal scale $\mathcal{L}$ of functions of $\mathcal{H}$ and any set function $F$ in $\mathcal{F}$, there is a finite or enumerable sequence $s_{1}, s_{2}, \ldots$ of distinct sections of $\mathcal{L}$, and a decomposition

$$
F=F^{(d)}+F_{2}^{\left(s_{1}\right)}+F_{3}^{\left(s_{1}\right)}+F_{2}^{\left(s_{2}\right)}+F_{3}^{\left(s_{2}\right)}+\ldots
$$

where $F^{(d)}$ has a diffuse $\mathcal{L}$-dimension spectrum, $F_{2}^{\left(s_{i}\right)}$ is strongly $s_{i}$-continuous and hypo $s_{i}$-singular, and $F_{3}^{\left(s_{i}\right)}$ is $s_{i}$-continuous and almost $s_{i}$-singular, for $i=1,2, \ldots$ The set of the sections $s_{i}$, and the decomposition (apart from the order of its terms) are uniquely determined by $\boldsymbol{F}$.

Proof. The result follows, mutatis mutandis, from the proof of Theorem 3 of [11]. The changes required are clear; in particular, it is necessary to work with the function $\left\|F_{2}^{(t)}\right\|+\left\|F_{3}^{(t)}\right\|$ of the section $t$ in $\mathcal{T}$ in place of the function $\left|F_{2}^{(\alpha)}\right|\left(I_{0}\right)$ of the real variable $\alpha$.

This theorem should be regarded as a more appropriate version of Theorem 6 of section 6 of [11]. In the earlier version, the components $F_{2}^{\left(s_{i}\right)}$ were lumped together with the component $F^{(d)}$, with the result that the component, said to have a diffuse $\mathcal{L}$-dimension spectrum, did not deserve this description.

The remainder of this paper will be concerned with the further study of the decomposition of (7). We shall investigate the types of sections $s$, for which the components $F_{2}^{(s)}$ and $F_{3}^{(s)}$ can be non-zero; we shall also show that, in certain circumstances, components of the decomposition are isolated in a certain sense.

\section{Sections in a scale of functions}

Let $\mathcal{L}$ be an irreducible maximal scale of functions of $\mathcal{H}$. Let $\mathcal{T}$ be the system of sections of $\mathcal{L}$. A set $Q$ of functions of $\mathcal{L}$ will be said to form a right basis for a section $s$ in $\mathcal{T}$, if $L_{s}$ is precisely the set of functions $h$ of $\mathcal{L}$, which satisfy $h \prec q$ for all $q$ in $Q$. Note that every section $s$ has the corresponding set $R_{s}$ as a right basis. Also, if a finite set of functions is a right basis for a section $s$, then the smallest of the functions of the set is thè minimal element of $R_{s}$, and the set containing just this one function is a right basis for $s$. Further, if a countable set, say $l_{1}, l_{2}, \ldots$, of functions of $\mathcal{L}$ forms a right basis for a section $s$ with no finite right basis, the sequence $u_{1}, u_{2}, \ldots$, given by

$$
u_{i}=\min \left\{l_{1}, l_{2}, \ldots, l_{i}\right\} \quad(i=1,2, \ldots),
$$


has no minimal element, and has a subsequence, $v_{1}, v_{2}, \ldots$ say, with

$$
v_{1} \succ v_{2} \succ \ldots
$$

forming a right basis for $s$. Thus there are three mutually exclusive possibilities:

(F) the section $s$ has a right basis consisting of a single function;

(E) the section $s$ does not have a finite right basis, but does have an enumerable right basis whose elements form a strictly monotonic sequence;

(N) the section $s$ has no enumerable right basis, but does have a non-denumerable right basis.

We can introduce a similar definition for a left basis, and make a similar classification, but, as $L_{s}$ has no maximal element, only the two cases (E) and (N) occur.

We say that a section $s$ is of type (AB), if its left hand bases fall under type (A) with $A=E$ or $N$, and its right hand bases fall under type (B) with $B=F, E$ or $N$. So a priori a section $s$ belongs to one of six types. But we shall soon eliminate two of these types.

First suppose that $s$ were a section of type (EF). Let $h$ be the minimal element of $R_{s}$ and let $v_{1}, v_{2}, \ldots$ be a left basis for $s$ with

$$
v_{1} \prec v_{2}<\ldots<h \text {. }
$$

By the remark after Theorem $A$, there is an element $k$ of $\mathcal{L}$ with

$$
v_{1} \prec v_{2} \prec \ldots \prec k \prec h \text {. }
$$

But now $k$ can belong neither to $L_{s}$ nor to $R_{s}$. This contradiction proves that there is no section of type (EF).

A precisely similar argument shows that there is no section of type (EE).

We are left with the sections of types (NF), (EN), (NE) and (NN); it is easy to construct examples of sections of each of these types. We now go on to discuss each of these types separately. However the types (NF) and (NN) have a property in common which has important consequences, and we consider this first. In both cases the section has no enumerable left basis and further $R_{s}^{\prime}$ has no countable cofinal sequence in $\mathcal{L}$, i.e. there is no countable sequence $r_{1}, r_{2}, \ldots$ in $\mathcal{L}$ with

$$
\ldots \prec r_{m} \prec \ldots \prec r_{2} \prec r_{1} \text {, }
$$

such that the members of $R_{s}^{\prime}$ are those $r$ in $\mathcal{L}$ such that $r_{m} \prec r$ for some integer $m$. This last property follows, in the case of a section of type (NF), by the argument used above to exclude sections of type (EF). 
We formulate the results for sections of these two types in terms of 'isolated components'.

DEFINITION. For any $s \in \mathcal{T}$, the component $F_{2}^{(s)}+F_{3}^{(s)}$ is said to be isolated, when there are sections $u, t$ of $\mathfrak{T}$ with $u<s<t$ such that, if $G$ is any set function of $\mathcal{F}$, which is u-continuous and $t$-singular and satisfies $|G| \leqslant|F|$, then $G$ is of exact $\mathcal{L}$-dimension $s$ and satisfies $|G| \leqslant\left|F_{2}^{(s)}+F_{3}^{(s)}\right|$.

LEMMA 1. Suppose $s$ is a section of $\mathcal{L}$ of type (NF) or (NN). Then the component $F_{2}^{(s)}+\bar{F}_{3}^{(s)}$ is isolated.

Proof. If $s$ is such a section, then we know there is no sequence $r_{1}, r_{2}, \ldots$, in $R_{s}^{\prime}$, such that any $h$ in $\mathcal{L}$ satisfying $h \prec r_{i}, i=1,2, \ldots$ cannot be in $R_{s}^{\prime}$; and there is no countable sequence $l_{1}, l_{2}, \ldots$ in $L_{s}$, such that any $h$ in $\mathcal{L}$ satisfying $l_{i} \prec h, i=1,2, \ldots$ cannot be in $L_{s}$. Under these circumstances, the remarks made in the proof of Theorem 2 apply, and there will be sections $l, r$ in $\mathcal{J}$ with

$$
l<s<r
$$

such that

$$
\begin{aligned}
& F_{1}^{(t)}=F_{1}^{(s)}+F_{2}^{(s)}+F_{3}^{(s)} \\
& F_{2}^{(t)}=0, \quad F_{3}^{(t)}=0, \quad F_{4}^{(t)}=F_{4}^{(s)} \quad \text { for } \quad l \leqslant t<s,
\end{aligned}
$$

and

$$
\begin{aligned}
& F_{1}^{(t)}=F_{1}^{(s)}, \quad F_{2}^{(t)}=0, \quad F_{3}^{(t)}=0, \\
& F_{4}^{(t)}=F_{2}^{(s)}+F_{3}^{(s)}+F_{4}^{(s)} \quad \text { for } \quad s<t \leqslant r .
\end{aligned}
$$

Consider any $G$ in $\mathcal{F}$, which is $l$-continuous and $r$-singular, and which satisfies $|G| \leqslant|F|$. Since $|G|$ is $l$-continuous, and

$$
|G| \leqslant|F|=\left|F_{1}^{(l)}+F_{2}^{(l)}+F_{3}^{(l)}\right|+\left|F_{4}^{(l)}\right|
$$

where $\left|F_{4}^{(l)}\right|$ is $l$-singular, it follows that

$$
|G| \leqslant\left|F_{1}^{(l)}+F_{2}^{(l)}+F_{3}^{(l)}\right|=\left|F_{1}^{(s)}\right|+\left|F_{2}^{(s)}+F_{3}^{(s)}\right| .
$$

Further, since $|G|$ is $r$-singular, and

$$
|G| \leqslant\left|F^{\prime}\right|=\left|F_{1}^{(r)}+F_{2}^{(r)}+F_{3}^{(r)}\right|+\left|F_{4}^{(r)}\right|
$$

where $\left|F_{1}^{(r)}+F_{2}^{(r)}+F_{3}^{(r)}\right|$ is $r$-continuous, it follows that

$$
|G| \leqslant\left|F_{4}^{(r)}\right|=\left|F_{2}^{(s)}+F_{3}^{(s)}\right|+\left|F_{4}^{(s)}\right| .
$$


Since $\left|F_{1}^{(s)}\right|$ and $\left|F_{4}^{(s)}\right|$ are complementary it follows from (8) and (9) that

$$
|G| \leqslant\left|F_{2}^{(s)}+F_{3}^{(s)}\right|
$$

This implies that $G$ is of exact $\mathcal{L}$-dimension $s$. Consequently the component $F_{2}^{(s)}+F_{3}^{(s)}$ is isolated.

\section{Sections of type (NF)}

Let $\mathcal{L}$ be an irreducible maximal scale of functions of $\mathcal{H}$ and let $s$ be a section of $\mathcal{L}$ of type $(\mathrm{NF})$. Let $h$ be the minimal element of $R_{s}$. Then $s$ is uniquely determined by $h$, and we write $s=s(h)$. We recall the definitions of the sets $\mathrm{C}(h), \mathrm{C}^{*}(h)$, $S(h)$ and $S^{*}(h)$ given in [1].

1. $\mathrm{C}(h)$ is the class of set functions $F$ of $\mathfrak{F}$, which are $h$-continuous; that is, those set functions such that $F(E)=0$, for any $E$ in $\mathcal{B}$ of zero $h$-measure.

2. $S(h)$ is the class of set functions $F$ of $\mathcal{F}$, which are $h$-singular; that is, those set functions, for which there is some $E_{0}$ in $\mathcal{B}$ with zero $h$-measure, such that

$$
F(E)=F\left(E \cap E_{0}\right) \text { for all } E \text { in } \mathcal{B}
$$

3. $\mathcal{C}^{*}(h)$ is the class of set functions $F$ of $\mathcal{F}$, which are strongly $h$-continuous; that is, those set functions such that $F(E)=0$, for any $E$ in $B$ of $\sigma$-finite $h$-measure.

4. $S^{*}(h)$ is the class of set functions $F$ of $\mathcal{F}$, which are almost $h$-singular; that is, those set functions, for which there is some $E_{0}$ in $B$ with $\sigma$-finite $h$-measure, such that (10) holds for all $E$ in $\mathcal{B}$.

In [11] we proved that $\mathcal{C}(h)$ and $S(h)$ and $\mathcal{C}^{*}(h)$ and $S^{*}(h)$ are pairs of complementary bands in $\mathcal{F}$. We remark here that the results could have been obtained a bit more directly, by use of results of Hahn and Rosenthal [6], rather than by use of the theory of bands.

In the special case, when $\mathcal{L}$ is dense, we can establish connections between the four bands associated with $h$ and the six bands associated with $s(h)$, by using the results of [13].

LEMMA 2. If $\mathcal{L}$ is dense, $h \in \mathcal{L}$ and $s(h)$ is the section of $\mathcal{L}$ of type $(\mathrm{NF})$ associated with $h$, we have

$$
\begin{gathered}
\mathcal{C}(h)=\mathrm{C}_{s(h)}, \quad \boldsymbol{S}(h)=\boldsymbol{S}_{s(h)}, \\
\mathcal{C}^{*}(h)=\mathcal{C}_{s(h)}^{*}=\mathcal{C}_{s(h)}^{* *}, \\
\boldsymbol{S}^{*}(h)=\boldsymbol{S}_{s(h)}^{*}=\boldsymbol{S}_{s(h)}^{* *}
\end{gathered}
$$


Proof. Comparing the definitions of $\mathcal{C}_{s(h)}$ and $\mathcal{C}(h)$, we see immediately that

$$
\mathcal{C}_{s(h)} \supset \mathcal{C}(h) \text {. }
$$

So consider any $F$ in $\mathcal{C}_{s(h)}$. Let $E$ be any set of $B$ with zero $h$-measure. Then, by a result of Besicovitch \{see Lemma 6 of $[13]$, there is a function $j$ of $\boldsymbol{H}$ with $j<h$ and $j-m(E)=\mathbf{0}$.

Since $\mathcal{L}$ is dense, it follows from $\left(\mathrm{P}_{6}\right)$ that there is a $g$ in $\mathcal{L}$ with $g<h j^{-1}$. Then, by $\left(\mathrm{P}_{3}\right)$, the function $l=h g^{-1}$ is in $\mathcal{L}$ and satisfies

$$
j \prec l \prec h \text {. }
$$

Thus we must have $l$ in $L_{s(h)}$, and

$$
l-m(E)=0 .
$$

As $F$ is in $C_{s(h)}$, this implies that $F(E)=0$. Hence $F$ is in $\mathrm{C}(h)$. Consequently

$$
\mathcal{C}_{s(h)}=\mathcal{C}(h) \text {. }
$$

It follows that the complementary bands $\boldsymbol{S}_{s(h)}$ and $\boldsymbol{S}(h)$ must coincide.

Comparing the definitions of $\mathcal{C}_{s(h)}^{*}, \mathcal{C}_{s(h)}^{* *}$ and $C^{*}(h)$, we see immediately that

$$
\mathcal{C}_{s(h)}^{*} \subset \mathcal{C}_{s(h)}^{* *} \subset \mathcal{C}^{*}(h) \text {. }
$$

Now consider any $F$ of $C^{*}(h)$. By Theorem 5 of [13], there is a function $g$ of $\mathcal{H}$ with $h \prec g$ and $F^{\prime} \in C^{*}(g)$. Since $\mathcal{L}$ is dense, it follows that there is an $l$ in $\mathcal{L}$ with

$$
h \prec l \prec g .
$$

Then $l \in R_{s(h)}^{\prime}$, and

$$
F \in C^{*}(l) \subset \mathcal{C}_{s(h)}^{* *} \subset \mathcal{C}_{s(h)}^{*} \text {. }
$$

Thus

$$
\mathrm{C}^{*}(h) \subset \mathrm{C}_{s(h)}^{* *} \subset \mathrm{C}_{s(h)}^{*} \subset \mathrm{C}^{*}(h) \text {. }
$$

Hence

$$
\mathcal{C}^{*}(h)=\mathcal{C}_{s(h)}^{*}=\mathcal{C}_{s(h)}^{* *}
$$

Consequently

$$
S^{*}(h)=S_{s(h)}^{*}=S_{s(h)}^{* *},
$$

as well. This completes the proof.

The following theorem provides considerable information about the components in Theorem 4 corresponding to sections of type (NF) in a dense scale.

TheOREM 5. Let $s$ be a section of type (NF) in a dense irreducible maximal scale $\mathcal{L}$, and let $h$ be the minimal element in $R_{s}$. Then, in the decomposition of a function $F$ of $\mathfrak{F}$, provided by Theorem 4 ,

$$
F_{2}^{(s)}=0
$$


$F_{3}^{(s)}$ is h-continuous and almost $h$-singular, and has a representation

$$
F_{3}^{(s)}(E)=\int_{E_{\mathrm{n} S}} f(x) d h-m(x)
$$

where $f(x)$ is a Borel-measurable point function which does not vanish on $S$, and $S$ is a Borel set of $\sigma$-finite h-measure. Further the component $F_{3}^{(s)}$ is isolated.

Proof. Using Lemma 2, we have

$$
F_{2}^{(s)} \in \mathcal{C}_{s}^{*}=C_{s}^{* *} \text { and } F_{2}^{(s)} \in S_{s}^{* *}
$$

Hence $F_{2}^{(s)}=0$.

Using Lemma 2 repeatedly, we have

$$
\begin{aligned}
F_{1}^{(s)}+F_{2}^{(s)} & \in \mathcal{C}_{s}^{*}=\mathcal{C}^{*}(h), \\
F_{3}^{(s)} \in \mathcal{C}_{s} \cap S_{s}^{*} & =\mathrm{C}(h) \cap S^{*}(h), \\
F_{4}^{(s)} \in S_{s} & =S(h) .
\end{aligned}
$$

So $F_{3}^{(s)}$ is $h$-continuous and almost $h$-singular. Further, comparing the decomposition

$$
F=F_{1}^{(s)}+F_{2}^{(s)}+F_{3}^{(s)}+F_{4}^{(s)}
$$

with that of Theorem 7 of [13], we identify $F_{3}^{(s)}$ with the second component of that theorem, so that it has a representation of the required form. Alternatively the representation may be obtained (as in Theorem 7 of [13]) by a direct application of the Radon-Nikodym theorem.

The fact that $F_{3}^{(s)}$ is isolated follows from Lemma 1 .

\section{Section of type (NE)}

Let $\mathcal{L}$ be an irreducible maximal scale of functions and let $s$ be a section of $\mathcal{L}$ of type (NE). In order to obtain significant results for sections of this type we shall have to assume that $\mathcal{L}$ is strongly dense. But first we prove a lemma.

LEMMA 3. Let $h_{1}, h_{2}, \ldots$ be a sequence of functions of $\mathcal{H}$ with

$$
h_{1} \succ h_{2} \succ \ldots
$$

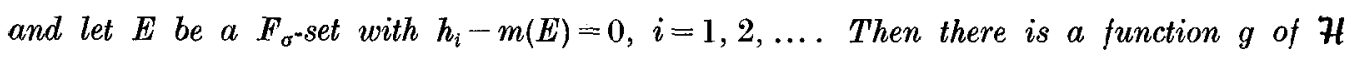
with $g \prec h_{i}, i=1,2, \ldots$, and $g-m(E)=0$. 
Proof. We first consider the case of a bounded closed set $E$. Since $E$ is compact, each covering of $E$ by open sets contains a finite covering of $E$. Since $h_{i}-m(E)=0$, for $i=1,2, \ldots$, it follows that for each pair of positive integers $i, j$ we can choose a finite system of open convex sets

$$
C_{1}^{(i, j)}, C_{2}^{(i, j)}, \ldots, C_{\mu}^{(i, j)}
$$

where $\mu=\mu(i, j)$, covering $E$, and such that

$$
\sum_{r=1}^{\mu} h_{i}\left(d\left(C_{r}^{(i . j)}\right)\right)<1 / j
$$

and

$$
\max _{1 \leqslant r \leqslant \mu} d\left(C_{r}^{(i, j)}\right)<1 / j
$$

Write

$$
d^{(i, j)}=\min _{1 \leqslant r \leqslant \mu} d\left(C_{r}^{(i, j)}\right)
$$

We now choose inductively a sequence of functions $k_{1}, k_{2}, \ldots$, a sequence of integers $j_{1}, j_{2}, \ldots$, and two sequences of real numbers $\xi_{1}, \xi_{2}, \ldots$, and $\eta_{1}, \eta_{2}, \ldots$, so that:

(1) $k_{1}(x)=h_{1}(x)$ for all $x \geqslant 0$, and $j_{1}=j(1)=1$;

(2) when $r \geqslant 1$ and $k_{r}(x)$ and $j_{r}=j(r)$ have been chosen, $\xi_{r}$ is chosen so that $h_{r+1}(x)>h_{r}(x)$ for all $x$ with $0<x<\xi_{r}$, and also $\xi_{r}<d^{(r, j(r))}$;

(3) when $r \geqslant 1$ and $k_{r}(x), j_{r}$ and $\xi_{r}$ have been chosen, $\eta_{r}$ is chosen, with $0<\eta_{r}<\xi_{r}$, so that $h_{r+1}\left(\eta_{r}\right)=h_{r}\left(\xi_{r}\right)$;

(4) when $r \geqslant 1$ and $k_{r}(x), j_{r}, \xi_{r}$ and $\eta_{r}$ have been chosen, $k_{r+1}(x)$ is defined by

$$
\begin{array}{lll}
k_{r+1}(x)=k_{r}(x) & \text { for } & \xi_{r} \leqslant x, \\
k_{r+1}(x)=k_{r}\left(\xi_{r}\right)=h_{r+1}\left(\eta_{r}\right) & \text { for } & \eta_{r} \leqslant x \leqslant \xi_{r}, \\
k_{r+1}(x)=h_{r+1}(x) & \text { for } & 0<x \leqslant \eta_{r} ;
\end{array}
$$

(5) when $r \geqslant 1$ and $k_{r}(x), j_{r}=j(r), \xi_{r}, \eta_{r}$ and $k_{r+1}(x)$ have been chosen, $j_{r+1}=j(r+1)$ is chosen to be an integer so large that

$$
\frac{1}{j_{r+1}}<\min \left(\eta_{r}, \frac{1}{r+1}\right)
$$

Consider the measure function $g(x)$ defined by

$$
g(x)=\lim _{r \rightarrow \infty} k_{r}(x)
$$

It is clear that

$$
g(x) \geqslant h_{r+1}(x) \text { for } 0<x<\xi_{r+1} \text {. }
$$


As $h_{r+1} \prec h_{r}$, it follows that $g \prec h_{r}$ for $r=1,2, \ldots$. Further, for each integer $r \geqslant 2$, the set of open convex sets

$$
C_{1}^{(r, j\langle r)\}}, \quad C_{2}^{(r, j, r))}, \ldots, C_{\mu}^{(r, j(r))}
$$

with $\mu=\mu(r, j(r))$, forms a covering of $E$ with

$$
\begin{aligned}
& \max _{1 \leqslant n \leqslant \mu} d\left(C_{n}^{(r, j(r))}\right)<\frac{1}{j(r)}<\eta_{r-1}, \\
& \min _{1 \leqslant n \leqslant \mu} d\left(C_{n}^{(r, j(r))}\right)=d^{(r, j(r))}>\xi_{r} .
\end{aligned}
$$

Thus $\quad \sum_{n=1}^{\mu} g\left(d\left(C_{n}^{(r, j(r))}\right)\right)=\sum_{n=1}^{\mu} k_{r}\left(d\left(C_{n}^{(r, j j(r))}\right)\right)=\sum_{n=1}^{\mu} h_{r}\left(d\left(C_{n}^{(r, j(r))}\right)\right)<1 / j(r)<1 / r$.

Hence $E$ is of zero $g$-measure.

Now suppose that $E$ is a $F_{\sigma}$-set. Then we have

$$
E=\sum_{j=1}^{\infty} \bigcup E_{j}
$$

for some sequence $E_{1}, E_{2}, \ldots$ of bounded closed sets.

As

$$
h_{i}-m\left(E_{j}\right) \leqslant h_{i}-m(E)=0,
$$

we can, for each $j$, choose a function $g_{j}$ of $\mathcal{H}$ with

$$
g_{j} \prec h_{i} \quad \text { for } i, j=1,2, \ldots
$$

and

$$
g_{j}-m\left(E_{j}\right)=0 \quad \text { for } j=1,2, \ldots
$$

Then, because $\mathcal{H}$ is strongly dense, we can choose a function $g$ of $\mathcal{H}$ with

$$
g_{j} \prec g \prec h_{i} \quad \text { for } i, j=1,2, \ldots
$$

Then

$$
g-m(E) \leqslant \sum_{j=1}^{\infty} g-m\left(E_{j}\right) \leqslant \sum_{j=1}^{\infty} g_{j}-m\left(E_{j}\right)=0 .
$$

This completes the proof.

Remark. The problem of extending this lemma to cover any Borel set $E$ seems to us to be open and interesting.

We can now easily prove

Leмma 4. If $\mathcal{L}$ is strongly dense, and $s$ is a section of $\mathcal{L}$ of type (NE), then

$$
\mathrm{C}_{s}=\mathrm{C}_{s}^{*}=\mathrm{C}_{s}^{* *}, \quad S_{s}=\boldsymbol{S}_{s}^{*}=S_{s}^{* *} .
$$


Proof. It suffices to prove that

$$
S_{s}=S_{s}^{*}=S_{s}^{* *}
$$

indeed, since

$$
S_{s} \subset S_{s}^{*} \subset S_{s}^{* *},
$$

it suffices to prove that

$$
S_{s}^{* *} \subset S_{s} .
$$

Consider then any function $F$ of $\mathcal{I}$ in $S_{s}^{* *}$. Let $v_{1}, v_{2}, \ldots$ be a right basis for $s$ with

$$
v_{1}>v_{2} \succ \ldots
$$

As $F \in S_{s}^{* *}$, we can choose sets $E_{1}, E_{2}, \ldots$ of $\mathcal{B}$, such that

$$
\begin{gathered}
F(E)=F\left(E \cap E_{i}\right) \quad \text { for all } E \text { of } \mathcal{B}, \\
v_{1}-m\left(E_{i}\right)=0,
\end{gathered}
$$

for $i=1,2, \ldots$. Then $E_{0}=\bigcap_{i=1}^{\infty} E_{i}$ is a set of $B$ with

$$
F(E)=F\left(E \cap E_{0}\right) \text { for all } E \text { of } \mathcal{B} \text {, }
$$

and

$$
v_{i}-m\left(E_{0}\right)=0 \quad(i=1,2, \ldots) .
$$

Since $F$ is an additive set function of $\mathcal{F}$, there is an $F_{\sigma^{-}}$-set $G$, contained in $E_{0}$, with

$$
|F|(G)=|F|\left(E_{0}\right)
$$

Since

$$
v_{i}-m(G)=0 \quad(i=1,2, \ldots)
$$

it follows from Lemma 3 , that there is a function $g$ of $\mathcal{H}$, with

$$
g \prec v_{i} \quad(i=1,2, \ldots),
$$

and

$$
g-m(G)=0
$$

Since $\mathcal{L}$ is strongly dense, there is an $l$ of $\mathcal{L}$ with

$$
g \prec l \prec v_{i} \quad(i=1,2, \ldots) .
$$

Since $v_{1}, v_{2}, \ldots$ is a right basis for $s$, it follows that $l \in L_{s}$. Now, we have $l \in L_{s}$,

$$
l-m(G) \leqslant g-m(G)=0,
$$

and, for each $E$ of $\mathcal{B}$,

$$
\begin{aligned}
|F(E \cap G)-F(E)| & \leqslant\left|F\left(E \cap E_{0}\right)-F(E)\right|+\left|F\left(E \cap E_{0}\right)-F(E \cap G)\right| \\
& \leqslant\left|F\left(E \cap E_{0}\right)-F(E)\right|+|| F\left|\left(E_{0}\right)-\right| F|(G)|=0 .
\end{aligned}
$$


Thus $F$ is concentrated on the set $G$ of $\mathcal{B}$, with zero $l$-measure, with $l$ in $L_{s}$, and so $F \in S_{s}$. Consequently

and the required result follows.

$$
S_{s}^{* *} \subset S_{s}
$$

As an immediate corollary of this lemma, we have

THEOREM 6. Let $s$ be a section of type (NE) in a strongly dense irreducible maximal

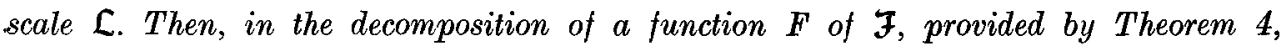

$$
F_{2}^{(s)}=F_{3}^{(s)}=0
$$

\section{Sections of type (EN)}

Let $\mathcal{L}$ be an irreducible maximal scale of functions and let $s$ be a section of $\mathcal{L}$ of type (EN). In order to obtain significant results for sections of this type, as for sections of type (NE) we shall have to assume that the scale $\mathcal{L}$ is strongly dense. But first we need two lemmas.

Our first lemma is a result which was proved, but not explicitly stated in [13] it introduces the concept of a set function $G$, which is uniformly $\operatorname{Lip} h(t)$, i.e. a set function $G$ of $\mathfrak{F}$, such that, for some constants $K$ and $\delta>0$, we have

$$
|G|(I) \leqslant K h(d(I))
$$

for all sub-intervals $I$ of $I_{0}$ with $d(I)<\delta$.

LEMMA 5. If $h \in \mathcal{H}$ and $F$ in $\mathcal{F}$ is h-continuous, then there is a sequence $E_{i}, i=1,2, \ldots$, of disjoint sets of $\mathcal{B}$, such that the set functions

$$
G_{i}(E)=F\left(E \cap E_{i}\right) \quad(i=1,2, \ldots),
$$

are each uniformly $\operatorname{Lip} h(t)$, and

$$
\begin{gathered}
F=\sum_{i=1}^{\infty} G_{i}, \\
\|F\|=\sum_{i=1}^{\infty}\left\|G_{i}\right\| .
\end{gathered}
$$

Proof. The result follow immediately, from part $(d)$ of the proof of Theorem 1 of [13], on taking

$$
E_{i}=K_{i}-K_{i-1} \quad(i=1,2, \ldots)
$$

LEMмA 6. Let $h_{1}, h_{2}, \ldots$ be a sequence of functions of $\mathcal{H}$ with

$$
h_{1}<h_{2}<\ldots,
$$

16-632918 Acta mathematica. 109. Imprimé 17 juin 1963. 
and let $F$ be a set function of $\mathcal{F}$, which is $h_{i}$-continuous for $i=1,2, \ldots$. Then there is a function $g$ of $\mathcal{H}$, with $h_{i} \prec g$ for $i=1,2, \ldots$, such that $F$ is still g-continuous.

Proof. Let $\varepsilon$ be given with $0<\varepsilon<1$. By Lemma 5, for each $j$, we can choose a. sequence $E_{i}^{(j)}, i=1,2, \ldots$ of disjoint sets of $\mathcal{B}$, such that the set functions

$$
G_{i}^{(j)}(E)=F\left(E \cap E_{i}^{(j)}\right) \quad(i=1,2, \ldots),
$$

are each uniformly Lip $h_{j}(t)$, and

$$
F=\sum_{i=1}^{\infty} G_{i}^{(j)}, \quad\|F\|=\sum_{i=1}^{\infty}\left\|G_{i}^{(j)}\right\|
$$

for $j=1,2, \ldots$. For each $j$, we choose an integer $k(j)$, such that

$$
\sum_{i=k(j) \div 1}^{\infty}\left\|G_{i}^{(j)}\right\|<\varepsilon^{j}
$$

and then write

$$
Q_{j}=\bigcup_{i=1}^{k(j)} E_{i}^{(j)}, \quad H_{j}=\sum_{i=1}^{k(j)} G_{i}^{(j)}
$$

Then $H_{j}$ belongs to $\mathcal{F}$, and is uniformly $\operatorname{Lip} h_{j}(t)$. Further

$$
\left\|F-H_{1}\right\|=\left\|\sum_{i=k(1)+1}^{\infty} G_{i}^{(1)}\right\|<\varepsilon
$$

and

$$
\left\|H_{j+1}-H_{j}\right\|=\left\|\sum_{i=k(j)+1}^{\infty} G_{i}^{(j)}-\sum_{i=k(j+1)+1}^{\infty} G_{i}^{(i+1)}\right\|<\varepsilon^{j}+\varepsilon^{j+1},
$$

for $j=1,2, \ldots$

$$
\text { Write } \quad K=\bigcap_{j=1}^{\infty} Q_{j}, \quad H=H_{1}+\sum_{j=1}^{\infty}\left\{H_{j+1}-H_{j}\right\} .
$$

Then $H$ belongs to $\mathcal{F}$, and satisfies

$$
H(E)=F(E \cap K),
$$

for all $E$ in $\mathcal{B}$. As $K \subset Q_{j}$, it follows that $H$ is uniformly $\operatorname{Lip} h_{j}(t)$, for $j=1,2, \ldots$ Also

$$
\|F-H\| \leqslant\left\|F-H_{1}\right\|+\sum_{j=1}^{\infty}\left\|H_{j+1}-H_{j}\right\|<\frac{2 \varepsilon}{1-\varepsilon} .
$$

Since $H$ is uniformly $\operatorname{Lip} h_{j}(t)$, for $j=1,2, \ldots$, and

$$
h_{1} \prec h_{2} \prec \ldots,
$$

we can use the usual technique to form a function $g$ of $\mathcal{H}$, with

$$
h_{1} \prec h_{2} \prec \ldots \prec g,
$$

such that $H$ is still uniformly $\operatorname{Lip} g(t)$. 
Repeating this construction, for different values of $\varepsilon$, we can choose a sequence $H_{1}, H_{2}, \ldots$ of functions of $\mathcal{F}$, with

$$
\left\|F-H_{j}\right\|<2^{-j} \quad(j=1,2, \ldots),
$$

and a sequence of functions $g_{1}, g_{2}, \ldots$ of $\mathcal{H}$, with

$$
h_{i} \prec g_{j} \quad \text { for } i, j=1,2, \ldots
$$

such that $H_{j}$ is uniformly $\operatorname{Lip} g_{j}(t)$, for $j=1,2, \ldots$. Now choose $g$ in $\mathcal{H}$, with

$$
h_{i} \prec g \prec g_{j} \quad \text { for } i, j=1,2, \ldots .
$$

Each function $H_{j}$ is uniformly $\operatorname{Lip} g(t)$. Further

$$
\boldsymbol{F}=H_{1}+\sum_{j=1}^{\infty}\left\{H_{j+1}-H_{j}\right\}
$$

with

$$
\sum_{j=1}^{\infty}\left\|H_{j+1}-H_{j}\right\|
$$

convergent. Hence, by Theorem 1 of [13], $F$ is $g$-continuous, as required.

LEMMA 7. If $\mathcal{L}$ is strongly dense, and $s$ is a section of $\mathcal{L}$ of type (EN), then

$$
\mathrm{C}_{s}=\mathrm{C}_{s}^{*}=\mathrm{C}_{s}^{* *}, \quad \mathrm{~S}_{s}=\mathrm{S}_{s}^{*}=\boldsymbol{S}_{s}^{* *}
$$

Proof. As

$$
\mathrm{C}_{s} \supset \mathrm{C}_{s}^{*} \supset \mathrm{C}_{s}^{* *}
$$

it suffices to prove that $\mathcal{C}_{s}^{* *} \supset \mathcal{C}_{s}$. Suppose then that $F$ is any set function of $\mathrm{C}_{s}$. Let $v_{1}, v_{2}, \ldots$ be a left basis for $s$ with

$$
v_{1} \prec v_{2} \prec \ldots
$$

Then $F$ is $v_{i}$-continuous, for $i=1,2, \ldots$ So, by Lemma 6 , there is a function $g$ of $\boldsymbol{H}$, with

$$
v_{i} \prec g \quad(i=1,2, \ldots),
$$

such that $F$ is $g$-continuous. Since $\mathcal{L}$ is strongly dense, there is a function $l$ of $\mathcal{L}$ with

$$
v_{i} \prec l \prec g \quad(i=1,2, \ldots) .
$$

As $s$ is of type (EN), the function $l$ belongs to $R_{s}^{\prime}$. Further $F$ is $l$-continuous. Thus $F$ is hyper $s$-continuous. This shows that $\mathcal{C}_{s} \subset C_{s}^{* *}$, and the proof is complete. 
Lemma 7 immediately gives

THEOREM 7. Let $s$ be a section of type (EN) in a strongly dense irreducible maximal scale $\mathcal{L}$. Then, in the decomposition of a function $F$ of $\mathcal{F}$, provided by Theorem 4 ,

$$
F_{2}^{(s)}=F_{3}^{(s)}=0
$$

This seems the most appropriate place to add a remark concerning the decomposition of Theorem 1 , when the section $s$ corresponds to a small generalized dimension. Since we have restricted our considerations to completely additive set functions on $I_{0}$, which are continuous, each $F$ of $\mathcal{F}$ is uniformly continuous in the sense that

$$
|F|(I) \rightarrow 0 \quad \text { as } \quad d(I) \rightarrow 0,
$$

where $I$ runs over all sub-intervals of $I_{0}$. Hence there is some function $h$ of $\mathcal{H}$, depending of $\boldsymbol{F}$, such that $F$ is uniformly lip $h(t)$. Hence $F$ is $h$-continuous. So, if $\mathcal{L}$ is dense, there is a section $l$ of $\mathcal{L}$ such that $F$ is $l$-continuous. Now we have

$$
F_{1}^{(t)}=F, \quad F_{2}^{(t)}=F_{3}^{(t)}=F_{4}^{(t)}=0,
$$

for all sections $t$ of $\mathcal{L}$ with $t<l$.

\section{Sections of type (NN)}

The components which Theorem 4 associates with a section of type (NN) are not accessible for study, and we know little about them. We shall give an example to show that these components can be non-zero; but first let us notice that Lemma 1 shows that such components are always isolated.

$A$ particular example. In [9] Rogers gave an explicit construction for a set function $P$ in the system $\mathcal{F}$, obtained by taking $k=1$ and $I_{0}$ to be the unit interval, and showed that it had certain properties which are relevant to the present work. To state the results we need to introduce the upper and lower classes associated with the law of the iterated logarithm.

For each $z$ with $0 \leqslant z<1$, let

$$
0 \cdot c_{1} c_{2} c_{3} \ldots
$$

be the binary decimal expansion of $z$, which does not terminate with 1 recurring, and let $S_{n}(z)$ denote the number of digits 1 among $c_{1}, c_{2}, \ldots c_{n}$. A real-valued continuous function $\phi(\lambda)$ is called a function of the lower class, if, for almost all $z$, we have

$$
S_{n}(z)<\frac{1}{2} n-\phi(n),
$$


for infinitely many values of $n$. Such a function $\phi(\lambda)$ is said to belong to the upper class, if, for almost all $z$, we have

$$
S_{n}(z)<\frac{1}{2} n-\phi(n)
$$

for at most a finite number of values of $n$. Each real-valued continuous function $\phi(\lambda)$ belongs to one or other of these classes. Using results of [12], Rogers proved that his function $P$ satisfies

ThEOREM H. Let $h \in \mathcal{H}$, and write

$$
\phi(\lambda)=\log _{2} 2^{3 \lambda / 2} h\left(2^{-3 \lambda}\right)
$$

If $\phi(\lambda)$ belongs to the upper class, then $P$ is strongly h-continuous; if $\phi(\lambda)$ belongs to the lower class, then $P$ is h-singular.

We now show that this implies

THEOREM 8. Let $\mathcal{L}$ be any irreducible maximal scale of functions of $\mathcal{H}$. Then the set $L$ of functions $h$ of $\mathcal{L}$ such that $\phi(\lambda)$, given by (11), belongs to the upper class, and the set $R$ of functions $h$ of $\mathcal{L}$ such that $\phi(\lambda)$ belongs to the lower class form the left and right classes of a section $s$ of $\mathcal{L}$, or this is the case after the maximal element of $L$ has been transferred to $R$, and in the decomposition of $F=P$, provided by Theorem 4 ,

$$
P=F_{2}^{(s)}+F_{3}^{(s)}
$$

If $\mathcal{L}$ is strongly dense, then $s$ is of type (NN).

Proof. Since the question of whether $\phi(\lambda)$ belongs to the upper or the lower class depends only on the behaviour of $h(t)$ for small positive values of $t$, it is easy to verify that $L$ and $R$ form the left and right classes of a section of $\mathcal{L}$, or that this is the case after the maximal element of $L$ has been transferred to $R$. Now Theorem $\mathrm{H}$ asserts that $P$ is $s$-continuous and hypo $s$-singular. Hence, in Theorem 4 ,

$$
P=F_{2}^{(s)}+F_{3}^{(s)}
$$

Now suppose that $\mathcal{L}$ is strongly dense. If $s$ were of type (NF) corresponding to a function $h$ of $\mathcal{L}$, then $h$ would belong to $L$ or $R$ and we would have a contradiction. Since $P$ is non-zero, it follows from Theorem 9 that $s$ cannot be of type (EN) or (NE). Hence $s$ is of type (NN). This can also be proved directly, by using the results of [12]. 


\section{The final decomposition theorem}

Combining the results of the previous sections we obtain the following decomposition theorem.

THEOREM 9. Given any strongly dense irreducible maximal scale $\mathcal{L}$ of functions of $\mathcal{H}$ and any set function $F$ in $\mathcal{F}$, there are finite or enumerable sequences $h_{1}, h_{2}, \ldots$ of functions of $\mathcal{L}$ and $s_{1}, s_{2}, \ldots$ of sections of type $(\mathrm{NN})$ of $\mathcal{L}$, and a decomposition

$$
\boldsymbol{F}=\boldsymbol{F}^{(d)}+\boldsymbol{F}_{0}^{\left(h_{1}\right)}+\boldsymbol{F}_{0}^{\left(h_{2}\right)}+\ldots+\boldsymbol{F}_{2}^{\left(s_{1}\right)}+\boldsymbol{F}_{3}^{\left(s_{1}\right)}+\boldsymbol{F}_{2}^{\left(s_{2}\right)}+\boldsymbol{F}_{3}^{\left(s_{2}\right)}+\ldots,
$$

where $F^{(d)}$ has a diffuse $\mathcal{L}$-dimension spectrum, $F_{0}^{\left(h_{i}\right)}$ is $h_{i}$-continuous and almost $h_{i}$-singular, and has a representation

$$
F_{0}^{\left(h_{i}\right)}(E)=\int_{E_{\mathrm{n}} S_{i}} f_{i}(x) d h_{i}-m(x)
$$

where $f_{i}(x)$ is a Borel-measurable point function which does not vanish on $S_{i}$, and $S_{i}$ is $a$ Borel set of $\sigma$-finite $h_{i}$-measure, for $i=1,2, \ldots$, and $F_{2}^{\left(s_{j}\right)}$ is strongly $s_{j}$-continuous and hypo $s_{j}$-singular, and $F_{3}^{\left(s_{j}\right)}$ is $s_{j}$-continuous and almost $s_{j}$-singular, for $j=1,2, \ldots$ The set of functions $h_{i}$, the set of sections $s_{j}$, and the decomposition (apart from the order of its terms) are uniquely determined by F. Further, each of the components

is isolated.

$$
\begin{gathered}
F_{0}^{\left(h_{i}\right)} \quad(i=1,2, \ldots), \\
F_{2}^{\left(s_{j}\right)}+F_{3}^{\left(s_{j}\right)} \quad(j=1,2, \ldots),
\end{gathered}
$$

We should perhaps remark at this stage that, although this theorem does not depend on the assumption of the Continuum Hypothesis, we have only been able to prove the existence of a strongly dense irreducible scale $\mathcal{L}$ of functions of $\mathcal{H}$, by assuming the truth of that hypothesis. Some further remarks concerning this theorem will be found in section 11 .

We were at one stage worried because the cardinality of the set $\mathcal{T}$ of sections of $\mathcal{L}$ must be $2^{\mathfrak{c}}$, and yet in a certain sense it was clear that not all of $\mathcal{J}$ could be relevant to the analysis of a single function $F$ in $\mathcal{F}$. Theorem 9 goes some way towards making this phenomenon explicit. For each $F$ in $\mathcal{F}$ there are "open intervals" in $\mathcal{J}$ in which $F$ has no contribution; these arise from each section $s(h)$ corresponding to an element $h$ in $\mathcal{L}$, and also from each section of type (NN), since even the null components $F_{2}^{(s)}+F_{3}^{(s)}$ are isolated. Thus for any particular $F$ in $\mathcal{F}$, not all of $\mathcal{T}$ is required for its complete analysis in the sense of Theorem 9 . 


\section{Epilogue}

This investigation has suggested a number of problems to us. We have already mentioned the problem of extending Lemma 3 to cover the case of a Borel set $E$. In our study of sections of type (EN) we were for a long time held up by our lack of a proof of a certain conjecture; eventually we found a way round the difficulty, using the results of [13]. One of us [10] subsequently proved the conjecture in the following form, which could be used in place of the results of [13] to prove Theorem 7 .

THEOREM. Let $E$ be a compact set in a separable metric space $\Omega$. Let $h_{1}, h_{2}, \ldots$ be functions of $\mathcal{H}$ with

$$
h_{1} \prec h_{2} \prec \ldots \prec h_{r} \prec \ldots
$$

Then, either (i) it is possible to express $E$ as $\bigcup_{r=1}^{\infty} E_{r}$ with

$$
h_{r}-m\left(E_{r}\right)=0 \quad(r=1,2, \ldots),
$$

or

(ii) there is an $h$ in $\mathcal{H}$ with

$$
h_{r} \prec h \quad(r=1,2, \ldots)
$$

and $E$ is of non- $\sigma$-finite $h$-measure.

This result has been extended to analytic sets $E$ by $\mathrm{M}$. Sion and D. Sjerve in $[14]$.

Theorems 8 and 9 leave open the problem: is it possible for each of the components $F_{2}^{(s)}, F_{3}^{(s)}$ associated with a section of type (NN) to be non-zero? Theorem 8 merely tells us that in the case of the set function $P$, one or other or both of these components is non-zero. We are tempted to believe that, even in the case of $P$, the component which is non-zero may depend on the choice of the strongly dense scale $\mathcal{L}$.

When we started this investigation, we hoped that it might lead to a decomposition of the type, provided by Theorem 9, but where each component, other than that with a diffuse dimension spectrum, would have an integral representation of the form (12). Now Theorem 8 shows that this is not possible, at any rate so long as our analysis is with respect to a scale of Hausdorff measures. It seems possible that this objective might still be reached, using an ordered system of uniform regular metric Carathéodory outer measures. But it begins to look as if it might be necessary to make the choice of the scale of measures dependent in some way on the function to be analysed. 
While it would be interesting to have theoretical methods of obtaining even more refined analyses of an additive set function, the present analysis is probably more than sufficiently refined for most particular set functions. It would be of interest to analyse some of the singular functions arising in number theory, and in the theory of probability, by the methods of this paper. It seems likely that in many cases it would be possible to choose a quite simple explicit scale $\mathcal{L}$, which would be sufficient to give a complete resolution into discrete components and a residuum with a diffuse dimension spectrum.

\section{References}

[1]. G. Birkhofr, Lattice theory. American Mathematical Society, Colloquium Publications, 25, New York, 1948.

[2]. S. Bochner \& R. S. Phillips, Additive set functions and vector lattices. Ann. of Math., 42 (1941), 316-324.

[3]. N. BourbakI, Les structures fondamentales de l'analyse, Book VI, Integration. Paris 1952 and 1956.

[4]. R. O. Davies, Non $\sigma$-finite closed subsets of analytic sets. Proc. Cambridge Philos. Soc., 52 (1956), 174-177.

[5]. P. Erdös, C. A. Rogers \& S. J. TAYLor, Scales of functions. J. Austral. Math. Soc., 1 (1960), 396-418.

[6]. H. Hahn \& A. Rosenthal, Set functions. Albuquerque, New Mexico, 1948.

[7]. F. RIEsz, Sur quelques notions fondamentales dans la théorie générale des opérations linéaires. Ann. of Math., (2), 41 (1960), 174-206.

[8]. C. A. Rogers, Dense scales of functions. J. Austral. Math. Soc., 2 (1961), 137-142.

[9]. —, Uniform set functions. Proc. Roy. Soc. A, 263 (1961), 149-160.

[10]. - Sets non- $\sigma$-finite for Hausdorff measures. Mathematika 9 (1962), 95-103.

[11]. C. A. Rogers \& S. J. TAYlor, Additive set functions in Euclidean space. Acta Math., 101 (1959). 273-302.

[12]. - - On the law of the iterated logarithm. J. London Math. Soc., 37 (1962), 145-151.

[13]. - - Functions continuous and singular with respect to a Hausdorff measure. Mathematika, 8 (1961), 1-31.

[14]. M. SION \& D. SJERvE, Approximation properties of measures generated by continuous set functions. Mathematika, 9 (1962), 145-156.

Received June 21, 1962 